\title{
The Differential Meaning of LV and LA Strains in Aortic Valve Stenosis: A Feature Tracking MRI Study
}

\author{
Jérôme Lamy ${ }^{1,2}$, Gilles Soulat ${ }^{4}$, Morgane Evin ${ }^{1,2}$, Khaoula Bouazizi-Verdier ${ }^{1,2}$, Alain Giron ${ }^{1}$, Alban \\ Redheuil $^{1,2,3}$, Elie Mousseaux ${ }^{4}$, Nadjia Kachenoura ${ }^{1,2}$ \\ ${ }^{1}$ Sorbonne Universités, UPMC Univ Paris 06, INSERM, CNRS, UMR 7371, UMRS 1146, \\ Laboratoire d'Imagerie Biomédicale, 75013, Paris, France \\ ${ }^{2}$ Institute of Cardiometabolism and Nutrition Imaging Core Lab, Paris, France \\ ${ }^{3}$ Department of Cardiovascular and Thoracic Imaging and Interventional Radiology, Institute of \\ Cardiology, Hôpital Pitié-Salpêtrière, Paris, France \\ ${ }^{4}$ Cardiology Department, European Hospital Georges Pompidou, Paris, France
}

\begin{abstract}
Feature tracking (FT) is an emerging approach for the evaluation of both left atrium (LA) and left ventricular $(L V)$ myocardial strain from cine MRI. We aimed to: 1$)$ perform a simultaneous evaluation of LA and LV strain in patients with aortic valve stenosis (AVS) and 2) evaluate associations of LA strain measurements with $L V$ remodeling (LVmass/LVend-diastolic volume) and strain.

We studied 55 patients (34 AVS:71 \pm llyears, 21 controls :66 \pm years) with preserved $L V$ ejection fraction $(L V E F)$. FT was applied to cine MRI to extract $L V$ longitudinal, circumferential and radial strain as well as LA longitudinal strain and radial motion fraction for the reservoir, conduit and LA contraction phases.

All LV strains decreased significantly in AVS. Similarly, all LA indices showed a decreasing trend in AVS patients as compared to controls. While reservoir and conduit phases LA strains were significantly associated with $L V$ remodeling and strain $(r>0.42)$, lower correlations were found for comparisons with LA contraction phase strains $(r<0.28)$. Such low association might be explained by the found bi-phasic distribution of contraction phase strains with $L V$ remodeling quartiles.

LA contraction strain seems to be a marker of intrinsic LA alteration that could be potentially used to predict progression towards atrial fibrillation in AVS patients.
\end{abstract}

\section{Introduction}

Aortic valve stenosis (AVS) is the most common valve disease in industrialized countries and its prevalence is unceasingly increasing with populations aging $[1,2]$. The reduction in aortic valve area in AVS patients leads to substantially increased left ventricular (LV) afterload inducing a significant increase in LV mass. Despite preserved LVEF in the early stage of AVS, the remodeled LV shows increased myocardial stiffness as well as elevated filling pressures. Consequently, left atrium (LA) will be exposed to such elevated pressures during diastole which would alter its function and dimensions, ultimately leading to atrial fibrillation (AF) [3].

While LV global longitudinal strain $(\mathrm{Sl})$ has been widely used in AVS populations, LA strain was studied in only few echocardiographic studies $[4,5]$ despite: 1$)$ the described pathophysiological coupling between LA and LV mechanics, 2) the LA strain incremental value in predicting AF occurrence in AVS patients undergoing aortic valve replacement [6]. Accordingly, it appears crucial to simultaneously evaluate LA and LV strain variations in AVS for a better understanding of changes in LA phasic function (reservoir, conduit, contraction) while considering their interactions with the wellestablished LV alterations.

Magnetic resonance imaging (MRI) is the reference modality for cardiac volumes and ventricular mass evaluation. In addition, its ability to estimate both LV and LA strain values using feature tracking (FT) on standard cine images, systematically acquired during MRI, has been demonstrated in several recent studies [7, 8]. MRI is therefore used on a group subjects including AVS and controls to: 1) perform a simultaneous evaluation of LA and LV strains and 2) evaluate associations of LA strain measurements with LV concentric remodeling.

\section{Material and methods}

\subsection{Population and data acquisition}

We studied 34 severe AVS patients $(71 \pm 11$ years, 11 
females) with preserved LVEF as well as 21 elderly controls (66 \pm 9 years, 8 females) undergoing a cardiac MRI exam on a 1.5 Tesla GE magnet. This study was approved by the local ethics committee and signed informed consent was obtained from all participants.

Preserved LVEF $(>50 \%)$ as well as severe AVS $\left(\mathrm{AVA}<1 \mathrm{~cm}^{2}\right)$ have been defined during screening at echocardiography prior to MRI exams.

Each subject had a conventional MRI including steady-state free precession (SSFP) acquisition in shortaxis, 4- and 2-chamber views. Such data were acquired during breath-hold, with the following scan parameters: acquisition matrix $=224 \times 192$, echo time $=1.63 \mathrm{~ms}$, repetition time $=3.65 \mathrm{~ms}$, flip angle $=50^{\circ}$, pixel size $=0.7 \times 0.7 \mathrm{~mm}^{2}$, slice thickness $=7$ to $8 \mathrm{~mm}$, inter-slice gap $=1 \mathrm{~mm}$. Short-axis acquisitions comprised 12 slices covering the whole ventricles and the number of phases per cardiac cycle was 40 phases for all views.

\subsection{Feature tracking algorithm}

The FT algorithm [7, 9] has been developed on Matlab (Mathworks, Natick, MA, USA) and is based on the interface between myocardium and the surrounding structures (blood, epicardial fat). First, endocardial contours were manually initialized on a single temporal phase corresponding to maximal cavity dilation and contrast was automatically enhanced. Epicardial contours were also initialized on the same phase for LV. The initialized points (20 markers) were then tracked forward and backward until the temporal phase used for initialization is reached.

For such tracking, a 10×10 mm region of interest is defined around each point of the initial contour. Then a map with cross-correlation values between this region and its spatial neighborhood on the neighboring phase is calculated. This cross-correlation map is weighted with additional maps derived from image properties and constraints related to physiological knowledge regarding contractile function. Such constraints maps comprised: 1) local differences in gray levels, 2) an elastic component used to preserve local spatiotemporal continuity of contour points displacement. Finally, to account for the periodicity of cardiac motion, myocardial contours were calculated by combining the temporal backward and forward tracking while using a sigmoid weighing to favor the tracking results closer to the initialization phase.

For all exams, the tracking process was applied to all views for LA and LV. No manual correction was performed at the end of the tracking.

\subsection{Indices of myocardial deformation}

The resulting FT contours were used to extract LV longitudinal, circumferential and radial strain curves as well as LA longitudinal strain and radial motion fraction curves, while considering reservoir, conduit and LA contraction phases. For LA radial motion fraction, endocardial movement towards the LA center of mass was considered.

Myocardial strain and motion fraction curves were used to automatically detect the following LV strain peaks: 1) LV longitudinal/circumferential strain ( $\mathrm{Sl} / \mathrm{Sc})$ systolic peak corresponding to the maximal shortening/contraction of the myocardium (Figure 1) and 2) LV radial strain $(\mathrm{Sr})$ systolic peak corresponding to the maximal myocardial thickening.

For the LA, peaks corresponding to the reservoir (s), conduit (e) and LA contraction (a) phases were derived from LA longitudinal strain $\left(\mathrm{Sl}_{\mathrm{s}}, \mathrm{Sl}_{\mathrm{e}}, \mathrm{Sl}_{\mathrm{a}}\right)$ and radial motion fraction $\left(\mathrm{Mr}_{\mathrm{s}}, \mathrm{Mr}_{\mathrm{e}}, \mathrm{Mr}_{\mathrm{a}}\right)$ curves (Figure 1).

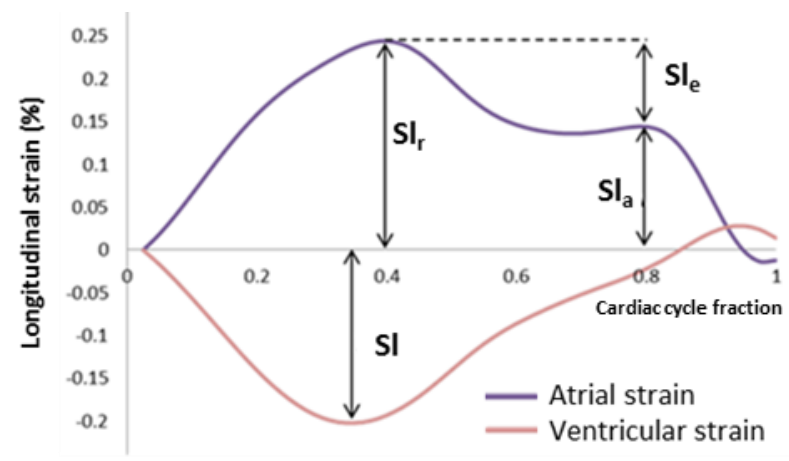

Figure 1. Longitudinal LA and LV strain curves and their extracted strain peaks: 1) $\mathrm{Sl}$ for $\mathrm{LV}, 2) \mathrm{Sl}_{\mathrm{s}}, \mathrm{Sl}_{\mathrm{e}}, \mathrm{Sl}_{\mathrm{a}}$ for $\mathrm{LA}$.

\subsection{Statistical analysis}

All continuous variables are reported as mean values \pm standard deviations. Strain values obtained for controls or patients were compared using a non-parametric Wilcoxon test. An ANOVA test was used to study LA strain parameters across LV remodeling quartiles subgroups defined on the entire population. Fisher's exact test was employed for non-continuous variable. A linear regression was used to study the associations of LA strains with $\mathrm{LV}$ remodeling and longitudinal strain. Pvalues $<0.05$ were considered as statistically significant. Statistical analysis was performed using the JMP-SAS software.

\section{Results}

\subsection{Population}

The FT tool performed successfully for all slices and the processing time for each subject (2-chamber, 4chamber, 5 to 6 short-axis slices) was around 10 minutes on a standard computer. Table 1 summarizes controls and patients basic characteristics, conventional MRI LV 
volumetric indices, as well as LV and LA strain indices.

Table 1. Subjects basic characteristics, conventional MRI LV indices and LA-LV strain indices

\begin{tabular}{|c|c|c|c|}
\hline & Controls (21) & AVS (34) & $\mathbf{p}$ \\
\hline Age (y) & $66 \pm 9$ & $71 \pm 11$ & 0.1363 \\
\hline Sex (females) & (8) & $(11)$ & 0.7727 \\
\hline $\operatorname{BSA}\left(\mathbf{m}^{2}\right)$ & $1.74 \pm 0.16$ & $1.88 \pm 0.21$ & 0.0149 \\
\hline \multicolumn{4}{|l|}{$\underline{L V \text { volumes and mass }}$} \\
\hline ESV/BSA $\left(\mathrm{mL} / \mathrm{m}^{2}\right)$ & $24 \pm 5$ & $27 \pm 11$ & 0.7290 \\
\hline $\operatorname{EDV} / \mathrm{BSA}\left(\mathrm{mL} / \mathrm{m}^{2}\right)$ & $68 \pm 13$ & $72 \pm 18$ & 0.5675 \\
\hline EF $(\%)$ & $65 \pm 4$ & $64 \pm 8$ & 0.7950 \\
\hline LVM (g) & $93 \pm 22$ & $160 \pm 40$ & $<.0001$ \\
\hline LVM/EDV (g/mL) & $0.79 \pm 0.18$ & $1.23 \pm 0.31$ & $<.0001$ \\
\hline LVM/BSA $\left(\mathrm{g} / \mathrm{m}^{2}\right)$ & $53 \pm 10$ & $85 \pm 19$ & $<.0001$ \\
\hline \multicolumn{4}{|l|}{$\underline{L V \text { strains }}$} \\
\hline SI (\%) & $-18.01 \pm 2.46$ & $-15.09 \pm 2.96$ & 0.0009 \\
\hline Sc $(\%)$ & $-19.33 \pm 2.99$ & $-17.13 \pm 3.35$ & 0.0193 \\
\hline $\operatorname{Sr}(\%)$ & $47.95 \pm 7.86$ & $39.01 \pm 14.21$ & 0.0059 \\
\hline \multicolumn{4}{|l|}{$\underline{L A \text { strains }}$} \\
\hline $\mathrm{Sl}_{\mathrm{s}}(\%)$ & $26.47 \pm 6.26$ & $21.89 \pm 6.96$ & 0.0049 \\
\hline $\mathrm{Sl}_{\mathrm{e}}(\%)$ & $12.13 \pm 4.86$ & $8.34 \pm 4.97$ & 0.0049 \\
\hline $\mathrm{Sl}_{\mathrm{a}}(\%)$ & $14.34 \pm 4.30$ & $13.55 \pm 4.26$ & 0.4724 \\
\hline $\mathrm{Mr}_{\mathrm{s}}(\%)$ & $25.89 \pm 5.15$ & $20.38 \pm 5.87$ & 0.0011 \\
\hline $\mathrm{Mr}_{\mathrm{e}}(\%)$ & $9.09 \pm 4.20$ & $6.72 \pm 4.98$ & 0.0387 \\
\hline $\mathrm{Mr}_{\mathrm{a}}(\%)$ & $16.80 \pm 4.45$ & $13.66 \pm 4.18$ & 0.0297 \\
\hline
\end{tabular}

No significant differences were noted between controls and AVS patients for indexed LV volumes and LVEF. As expected, LVM and LV remodeling index (LVM/EDV) were significantly higher in AVS patients as compared to controls. Furthermore, LA and LV strain indices showed a drop in magnitude in AVS patients as compared to controls, which reached significance for all indices except for the LA longitudinal strain corresponding to the contraction phase $\left(\mathrm{Sl}_{\mathrm{a}}\right)$.

\subsection{Interaction of $L A$ strain indices with LV remodeling and strain}

Results of linear regression between LA strain indices and the well-established LV remodeling (LVM/EDV) and longitudinal strain indices ( $\mathrm{Sl}$ ) are summarized in Table 2.

Table 2. LA strain measurements and their associations with LV remodeling and longitudinal strain (Sl).

\begin{tabular}{lcccc} 
& \multicolumn{2}{c}{ LVM/EDV } & \multicolumn{2}{c}{ Sl } \\
& $\mathbf{r}$ & $\mathbf{p}$ & $\mathbf{r}$ & $\mathbf{p}$ \\
\hline $\mathbf{S l}_{\mathbf{s}}$ & 0.42 & $\mathbf{0 . 0 0 1 5}$ & 0.52 & $<.0001$ \\
$\mathbf{S l}_{\mathbf{e}}$ & 0.54 & $\mathbf{< . 0 0 0 1}$ & 0.61 & $<.0001$ \\
$\mathbf{S l}_{\mathbf{a}}$ & 0.03 & 0.8480 & 0.10 & 0.5446 \\
$\mathbf{M r}_{\mathbf{s}}$ & 0.52 & $<.0001$ & 0.57 & $<.0001$ \\
$\mathbf{M r}_{\mathbf{e}}$ & 0.49 & $\mathbf{0 . 0 0 0 2}$ & 0.54 & $<.0001$ \\
$\mathbf{M r}_{\mathbf{a}}$ & 0.26 & 0.0571 & 0.28 & $\mathbf{0 . 0 3 7 2}$ \\
\hline
\end{tabular}

LA radial and longitudinal indices for reservoir and conduit phases were significantly associated with LV parameters. Associations with LA contraction phase indices resulted in the lowest correlation coefficients with both LV remodeling and longitudinal strain.

Table 3 summarizes distribution of LA strain indices values across LV remodeling quartiles. While LA indices corresponding to the reservoir and conduit phases decreased gradually across LV remodeling quartiles, LA contraction indices showed a bi-phasic variation profile. Indeed, LA contraction strain indices showed and increasing trend between quartile 1 and quartiles 2 and 3 , and then a decreasing trend towards quartile 4 (Figure 2).

Table 3. LA strain indices distribution across LV remodeling quartiles. P-values for ANOVA test across quartiles group are provided.

\begin{tabular}{lccccc}
\hline & Quart.1 & Quart.2 & Quart.3 & Quart.4 & p \\
\hline $\mathbf{L V M} / \mathbf{E D}$ & $0.69 \pm 0.12$ & $0.89 \pm 0.07$ & $1.16 \pm 0.09$ & $1.54 \pm 0.23$ & $<.0001$ \\
$\mathbf{V}$ & & & & & \\
$\mathbf{S l}_{\mathbf{s}}$ & $27.52 \pm 8.67$ & $23.81 \pm 4.26$ & $23.57 \pm 7.14$ & $19.51 \pm 5.13$ & $\mathbf{. 0 2 7 2}$ \\
$\mathbf{S l}_{\mathbf{e}}$ & $14.33 \pm 5.58$ & $9.65 \pm 3.21$ & $8.92 \pm 4.55$ & $6.09 \pm 3.80$ & $\mathbf{. 0 0 0 2}$ \\
$\mathbf{S l}_{\mathbf{a}}$ & $13.19 \pm 5.71$ & $14.16 \pm 2.64$ & $14.66 \pm 3.95$ & $13.42 \pm 4.36$ & .7994 \\
$\mathbf{M r}_{\mathbf{s}}$ & $26.24 \pm 7.44$ & $23.59 \pm 5.22$ & $22.25 \pm 4.89$ & $17.75 \pm 3.64$ & $\mathbf{. 0 0 2 3}$ \\
$\mathbf{M r}_{\mathbf{e}}$ & $11.27 \pm 3.77$ & $7.51 \pm 3.31$ & $6.54 \pm 3.84$ & $5.05 \pm 3.34$ & $\mathbf{. 0 0 0 3}$ \\
$\mathbf{M r}_{\mathbf{a}}$ & $14.97 \pm 5.47$ & $16.07 \pm 3.58$ & $15.71 \pm 4.41$ & $12.70 \pm 4.06$ & .2234 \\
\hline
\end{tabular}

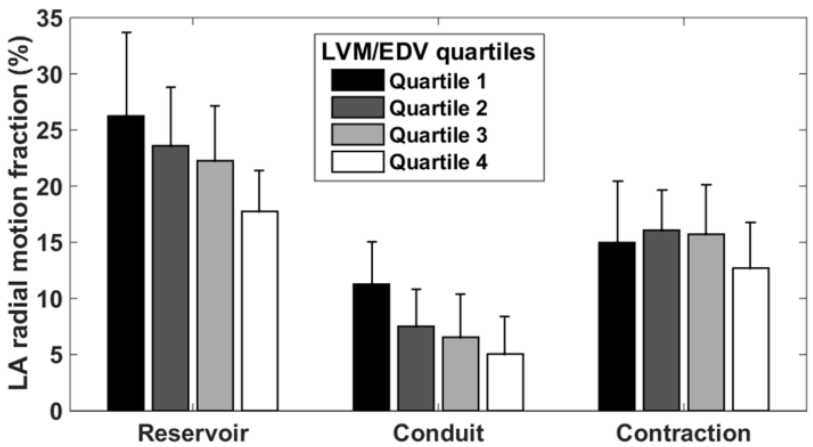

Figure 2. LA radial motion fraction measurements across concentric remodeling quartiles groups for the $3 \mathrm{LA}$ phases.

\section{Discussion and conclusion}

Accurate evaluation of the cardiac impairment is crucial for clinical management of patients with AVS. To this end biomarkers such as aortic valve area and LV concentric remodeling have been proposed. While these indices allow, respectively, evaluating the extent of valve area reduction and subsequent $\mathrm{LV}$ remodeling caused by pressure overload, the extent of LA alteration and its interaction with LV has been quantified only in few studies. Furthermore, it is known that LV remodeling and its loss of compliance and relaxation capacities are strongly related to LA impairment leading ultimately to atrial fibrillation.

Accordingly FT has been used in this study on standard cine MRI of patients with AVS and age equivalent healthy controls for a simultaneous functional evaluation of LV and LA and we found that: 1) despite a preserved LVEF in both groups, strain indices were 
reduced in AVS as compared to controls in both $\mathrm{LV}$ and LA consistently with previous findings [10], 2) LA strain indices of the reservoir and conduit phases were strongly associated with both LV remolding and LV longitudinal strain, while LA strain indices corresponding to the contraction phase resulted in lower to non-significant correlations with LV indices, 3) while LA indices corresponding to the reservoir and conduit phases decreased gradually across LV remodeling quartiles, LA contraction indices showed a bi-phasic variation profile.

The differential association of the two first LA phases (reservoir and conduit) and LA contraction phase with LV remodeling and longitudinal strain can be explained by the fact that reservoir and conduit phases are strongly associated to $\mathrm{LV}$ indices because of the mechanistic interaction between LA and LV during LV contraction and relaxation. Indeed the mitral descent during $\mathrm{LV}$ contraction would stretch the atrium and affect its myocardial strain values [11]. Besides, appropriate LV remodeling and longitudinal strain being markers of appropriate LV compliance, would be associated to LV relaxation capacities during early diastole and accordingly to the LA conduit phase indices. Finally, the poor association between LV indices and LA contraction might be explained by the absence of LV motion during this phase but also by the fact that LA contraction shows a biphasic behavior according to the LV remodeling process. Indeed, LA contraction first increased between quartiles 1 and 3 of LV remodeling indicating the LA compensatory behavior to impaired early LV filling, before dropping towards quartile 4 indicating a loss in LA contractility.

Associations of LA function with LV remodeling and LV longitudinal strain revealed that: 1) LA reservoir function seems to be a mixture of LA compliance impairment due to fibro-fatty infiltration in the LA myocardium as well as of LV longitudinal dysfunction. Indeed in the present study LA reservoir function seems to be related to $\mathrm{LV}$ longitudinal strain while it has been significantly associated to histological LA tissue characteristics in a previous study [12], 2) LA conduit phase might be mainly LV driven, 3) LA contraction phase strain indices were not linearly associated to LV remodeling but rather in a bi-phasic behavior indicating its ability to reflect the compensatory role of the LA to LV early filling but also the loss in LA myocardium contractility. Accordingly, while LA reservoir and conduit strains can be regarded as partially LV-driven indices, LA contraction strain can be regarded as an intrinsic LA function index.

\section{Acknowledgements}

$\mathrm{JL}$ and ME received a research grant from the Institute of Cardio-metabolism and nutrition (ICAN). This work was funded by Assistance Publique-Hôpitaux de Paris (grant PHRC RACINE) and the authors were totally independent of the funder for all scientific aspects of this research. The authors would like to thank Juliette DjadiPrat and Valentina Zhygalina from the Clinical Research Unit of the European Georges Pompidou Hospital for their help in this study. The authors have no conflicts of interest and nothing to disclose.

\section{References}

[1] Iung B, Vahanian A. Epidemiology of Acquired Valvular Heart Disease. Can J Cardiol 2014; 30: 962-970.

[2] Rajamannan NM, Bonow RO, Rahimtoola SH. Calcific aortic stenosis: an update. Nat Rev Cardiol 2007; 4: 254 262.

[3] Bonow RO, Carabello BA, Chatterjee $\mathrm{K}$, et al. ACC/AHA 2006 Guidelines for the Management of Patients With Valvular Heart Disease. Circulation 2006; 114: e84-e231.

[4] O'Connor K, Magne J, Rosca M, et al. Impact of Aortic Valve Stenosis on Left Atrial Phasic Function. Am J Cardiol 2010; 106: 1157-1162.

[5] Salas-Pacheco JL, Ávila-Vanzzini N, Eugenia R-EM, et al. Left atrium function by $2 \mathrm{D}$ speckle tracking in aortic valve disease. Echocardiography 2016; 33: 1828-1834.

[6] Cameli M, Lisi M, Reccia R, et al. Pre-operative left atrial strain predicts post-operative atrial fibrillation in patients undergoing aortic valve replacement for aortic stenosis. Int J Cardiovasc Imaging 2014; 30: 279-286.

[7] Evin M, Cluzel P, Lamy J, et al. Assessment of left atrial function by MRI myocardial feature tracking. J Magn Reson Imaging JMRI 2015; 42: 379-389.

[8] Andre F, Steen H, Matheis P, et al. Age- and genderrelated normal left ventricular deformation assessed by cardiovascular magnetic resonance feature tracking. $J$ Cardiovasc Magn Reson Off J Soc Cardiovasc Magn Reson 2015; 17: 25.

[9] Evin M, Redheuil A, Soulat G, et al. Left atrial aging: a cardiac magnetic resonance feature-tracking study. Am J Physiol Heart Circ Physiol 2016; 310: H542-549.

[10] O'Connor K, Magne J, Rosca M, et al. Left atrial function and remodelling in aortic stenosis. Eur $J$ Echocardiogr 2011; 12: 299-305.

[11] Barbier P, Solomon SB, Schiller NB, et al. Left Atrial Relaxation and Left Ventricular Systolic Function Determine Left Atrial Reservoir Function. Circulation 1999; 100: 427-436.

[12] Huber A, Lamy J, Rahhal A, et al. Cardiac MR strain: a noninvasive biomarker of fibro-fatty remodeling of the left atrial myocardium. Radiology.

Address for correspondence.

Jérôme Lamy

Faculté de Médecine Pierre et Marie Curie - Site PitiéSalpêtrière- LIB, 91 Bd de l'Hôpital, 75013, Paris, France 